\title{
Análisis del consumo máximo de oxígeno (vo2máx) post intervención de un programa en juegos pre-deportivos.
}

Analysis of the maximum oxygen consumption (VO2max) post-intervention of a program in pre-games sports.

Julio Alfonso Mocha Bonilla. ${ }^{1}$, Santiago Ernesto Garcés Duran. ${ }^{2}$, Segundo Víctor Medina Paredes. ${ }^{3}$, Washington Ernesto Castro Acosta. ${ }^{4}$, Mayra Gabriela Suntasig Muso. ${ }^{5}$

\begin{abstract}
.
DOI: https://doi.org/10.33262/cienciadigital.v2i2.85

The present investigation is focused on the effect the application. It is concerning about an intervention program to analyze the maximum oxygen consumption in a sample of adolescents from 17 to 19 years. It is in order to observe the physical condition of the people. The methodology responds to a quasi-experimental design, with is the application of an intervention based on a pre-sports games program. It is established by taking data before and after the application of the program with the use of the Course Navette Test. It measures the maximum oxygen consumption in each one of the participants. The program of pre-sports games was applied during the classes of Physical Education. It had a duration of six months. The results showed significant advances after the application of the program which allowed us to conclude that our experiment had obtained scores over VO2max after the application of this program. It had improved the physical condition in the study subjects
\end{abstract}

Keywords: Vo2máx, Pre-Deportive Games, Test Course Navette, Increase in Speed.

${ }^{1}$ Universidad Técnica de Ambato, FCHE, Ambato, Ecuador, ja.mocha@uta.edu.ec

${ }^{2}$ Universidad Técnica de Ambato, FCHE, Ambato, Ecuador, se.garces@uta.edu.ec

${ }^{3}$ Universidad Técnica de Ambato, FCHE, Ambato, Ecuador, sv.medina@uta.edu.ec

${ }^{4}$ Universidad Técnica de Ambato, FCHE, Ambato, Ecuador, we.castro@uta.edu.ec

${ }^{5}$ Universidad Técnica de Ambato, FCHE, Ambato, Ecuador, msuntasig8299@uta.edu.ec 


\section{Resumen.}

La presente investigación se focalizó en el efecto de la aplicación de un programa de intervención para analizar el consumo de oxígeno máximo en una muestra de adolescentes de 17 a 19 años, con la finalidad de observar la condición física. La metodología responde a un diseño cuasi experimental, es decir, la aplicación de una intervención basada en un programa de juegos pre-deportivos establecida mediante la toma de datos antes y después de la aplicación del programa, con la utilización de test de Course Navette, el cual mide el consumo de oxígeno máximo en cada uno de los participantes; el programa de juegos pre-deportivos se aplicó durante las clases de Educación Física, cuya duración fue de seis meses. Los resultados muestran avances significativos luego de la aplicación del programa, lo que lleva a concluir que, nuestro experimento obtuvo mejores puntuaciones sobre el VO2max tras la aplicación del programa de intervención, lo cual mejoró la condición física en los sujetos de estudio.

Palabras Claves: VO2máx, juegos pre-deportivos, Test Course Navette, condición física.

\section{Introducción.}

Los juegos pre-deportivos son actividades lúdicas que ayudan al desarrollo del niño y el joven, es decir, un adecuado aprendizaje motor, una alternativa pedagógica (ArévaloSánchez, 2016) fundamentada en la iniciación deportiva, especialmente en el atletismo (Yanelis Cobas-Ortiz, 2015), suele ser una estrategia efectiva dentro del proceso de preparación deportiva para el desarrollo de las capacidades y habilidades motrices (Valarezo Mendoza, 2017). Cuando se habla de la modalidad de enseñanza y aprendizaje, por parte de los docentes de Educación Física, se puede mencionar que este proceso desde la antigüedad se basó en un estilo de enseñanza tradicional (Delgado Noguera, 2015) pues los maestros no eran especializados y no buscaban un método divertido y significativo mediante la utilización de técnicas para la enseñanza de las carreras de resistencia.

En el Ecuador, la información sobre la práctica de los juegos pre-deportivos, específicamente para mejorar la condición física, inician con la implementación de una nueva reforma educativa curricular de Educación Física (Cabrera Hernández \& Cruz Vadillo, 2016), la cual toma en cuenta las necesidades que requieren, tanto los educandos como los educadores, para que las clases de Educación Física sean de gran interés y disfrute personal (Yépez, M. M. M., \& Benítez, J. E. M, 2017), pues hace dos décadas, el profesor se limitaba a llevar una planificación de clase muy ambigua, que no daba ningún resultado en la iniciación deportiva del atletismo, que contribuya en el fortalecimiento de la condición física de sus estudiantes. 
En los actuales momentos, en las instituciones educativas, existen docentes que poseen conocimiento de las actividades físico deportivas, y han sido preparados para trabajar con personas en el ámbito deportivo; al utilizar juegos pre-deportivos dentro de sus planificaciones diarias de clase, es decir, preparan las actividades deportivas con una noción de enseñanza lúdica, para mejorar la condición física de las personas, tomando así una decisión en la práctica educativa y deportiva (González, 2016).

Aplicar los juegos pre-deportivos en las clases permite que los jóvenes se sientan libres y mantengan una condición física estable, mediante actividades lúdicas y recreativas; por consiguiente, la actividad física, mediante los juegos pre-deportivos practicadas en los recreos y las clases de Educación Física, promueven efectos en la composición corporal y la condición física, al tener como resultados, un mejor estilo de vida, condición física optima y una masa corporal normal (Pumar, 2015); como consecuencia, existe una mejora en la motricidad gruesa a través de la aplicación de los juegos lúdicos (Ruitón, 2015).

Los espacios de entretenimiento para los niños y jóvenes deberían ser un lugar de convivencia y de desarrollo lúdico, tomando al juego como una manifestación de apoyo, enseñanza y desarrollo en cada individuo, es una manera lúdica de interactuar con los grupos sociales para mitigar los conflictos en los espacios de entretenimiento (Graña, 2015). Los juegos pre-deportivos suelen ser una estrategia pedagógica para trabajar en el campo educativo-deportivo, pues contribuyen en la mejora de las habilidades y destrezas motoras básicas y específicas (Granja, 2016).

La práctica de los juegos deportivos favorece el estado de anímico y el equilibrio emocional de los participantes (Díaz R. M., 2016), elementos que suelen ser imprescindibles dentro de los planes de clase de Educación Física y, sobre todo, en el deporte, ya que al practicar los juegos pre-deportivos se permite la influencia positiva dentro del rendimiento técnico, táctico y motriz en los deportes (Morales, 2017).

En las escuelas y colegios, el profesorado observa, por medio del juego, la interrelación de los individuos, su manera de actuar, pensar, e interrelacionarse, funciones importantes del juego en el desarrollo del ser humano (Gómez, 2017). En definitiva, las actividades lúdicas son aspectos fundamentales en el desarrollo, tanto físico como cognitivo, que contribuye a la mejora de las habilidades y destrezas (Miranda, 2016) por consiguiente el objetivo de nuestro estudio es valorar el Consumo Máximo de Oxigeno antes y después de un programa de intervención de juegos pre-deportivos.

\section{Revisión de la literatura.}

Estudios para mantener una condición de salud estable, tomando en cuenta la capacidad aeróbica máxima y la calidad de vida, mediante el test de Course Navette y la escala de KIDSCREEN, muestran que los escolares que tienen un nivel mayor de VO2max, tienen una mejor calidad de vida (Casas, 2015). También, se ha utilizado un test de campo, 
conocido como carrera progresiva o entrenamiento aeróbico, el cual permite conocer el tiempo límite de (velocidad), además se aplicó el test de Leger para medir el VAM y el tiempo límite, se concluyó no encontrar relación entre la velocidad aeróbica máxima y el tiempo límite (Díaz D. A., 2015). Por otra parte, en una evaluación realizada a estudiantes mujeres y hombres de la Universidad Veracruzana de la Carrera de Educación Física Deporte y Recreación, participantes voluntarios, se estableció la estimación del consumo de oxigeno máximo (Figueroa, 2015).

Al investigar los niveles de VO2 máximo, en futbolistas adolescentes, caracterizando el comportamiento del consumo de oxígeno en diferentes altitudes, se experimentó sobre la compensación deportiva, para esto se utilizó el Yoyo test, mismo que sirvió para medir el VO2 Max y el ácido láctico en la sangre, con lo cual se observó si el lugar de entrenamiento afecta o no el perfeccionamiento del futbolista (Ruíz, 2015). También se utiliza el espirómetro para deducir el consumo de VO2max en los segmentos corporales y evaluar el resultado que da el corazón, es decir, la relación entre la FC y VO2max (Velásquez, 2015).

Como consecuencia de aplicar un programa de alta intensidad sobre el consumo de oxígeno máximo y el recobro de la respiración en nadadores jóvenes, se evaluó el VO2max, utilizando el test de Navette y el Yoyo test, se obtuvo como resultado mejoras en el consumo de oxígeno máximo, y la recuperación cardiorrespiratoria después de la intervención (Véliz, 2016).

Los diferentes estudios reportan coherencia en cuanto a la valoración del VO2max entre el Yoyo test, y la medición directa de la condición física, examinando el grado de correlación estimado, que presentan los dos test, por consiguiente, se hace necesario meditar las pruebas que se deben aplicar para evaluar la condición del deportista (Longo, 2017).

Los diversos rastreadores de actividad física nos lleva a utilizar una nueva característica conocida como el Fitbit Charge 2, "Cardio Fitness Level", el cual es comparable con las puntuaciones de VO2max, pues permite conocer la condición física aeróbica de las personas, sin embargo las puntaciones de VO2 dadas por Fitbit Charge 2, no siempre coincidían con los valores dados por el carro metabólico, de aquí la importancia de tomar el test en la práctica misma, con lo cual se puede observar cada una de las fases establecidas en los protocolos (Lucio, 2018); en definitiva, se menciona que el VO2max es uno de los mejores indicadores para realizar estudios en la preparación física (Polo-Benítez, 2017). Luego del análisis de la literatura de soporte, el grupo de investigadores decidió aplicar el test de Course Navette como el instrumento adecuado para medir el VO2max, por la realidad de los participantes en el estudio.

\section{Metodología.}


Sujetos: se tomó una muestra de estudio, misma que estuvo conformada por 90 estudiantes de tercer año de Bachillerato General Unificado, en un rango de edad comprendida entre los 17 a 19 años; se realizó un criterio de exclusión de 13 sujetos por presentar problemas fisiológicos en su condición física, quedando una muestra de 77 participantes.

Protocolos: en primer lugar, se ejecutó la revisión de la literatura sobre el objetivo del estudio, luego se procedió con el trabajo de campo, empleando la aplicación del test de course navette en un pre test (antes de la aplicación del programa de intervención), permitiendo así una estimación de resultados para diseñar un programa adecuado que mejore la condición física; posteriormente, se realizó el programa de juegos pre-deportivos planificados y ejecutados durante las horas de educación física, por el tiempo estimado de seis meses, con una ejecución de seis horas semanales, tres veces a la semana.

Procedimiento: Con los estudiantes se procedió a la aplicación del test; la colaboración de los involucrados fue presta y voluntaria, a su vez, se socializó el contenido y características del test de Course Navette, ya que se requería conocer si existen estudiantes que padecen alguna enfermedad cardiorrespiratoria, pues, de ser el caso, no pueden realizar el test, para evitar problemas de salud; por tanto, el test ayudó a conocer en qué estado físico se encuentran los estudiantes y como se encuentra su VO2máx; el proceso se ejecutó con los requerimientos planificados tanto en el pre y post test aplicado durante las clases de Educación Física, con los docentes especialistas del área.

La primera toma del pre test se realizó en el mes de noviembre del período escolar 2017 2018, posteriormente, se procedió a la segunda toma del post test, luego de seis meses de trabajo en el mes de mayo, procediendo a evaluar el incremento en el consumo de oxígeno, realizando el análisis y la comparación entre los dos resultados para verificar si hubo o no mejoría en los sujetos de estudio. García (2014) considera que para la aplicación del test se debe contar con un test audible, delimitar una distancia entre 2 líneas separadas por 20m en doble sentido, ida y vuelta; además se debe instalar un reproductor de audio a un costado del espacio para que se pueda escuchar la señal sonora, para dar inicio al test y obtener una correcta ejecución.

Instrumentos: Los estudiantes fueron sometidos a una prueba física denominada test de Course Navette, utilizado para determinar la capacidad aeróbica en personas jóvenes con un grado de entrenamiento medio o bajo; a través de los resultados que se obtienen de la prueba, lo cual nos permite evaluar el consumo máximo de oxígeno en cada individuo, atendiendo a la velocidad que cada persona pueda realizar en su último recorrido durante el test (Martinez López, 2004). 
La información fue procesada utilizando el software estadístico SPSS versión 21 para comparar el antes y después de la ejecución del programa de intervención, utilizando la prueba estadística de kolmogorov - smirnov para una muestra, y comprobar la hipótesis de trabajo.

\section{Resultados.}

A continuación, se pueden observar los resultados obtenidos de la aplicación del (pre test) según las tablas y figuras estadísticas realizadas.

\section{Resultados de la aplicación del pre test (course navette)}

Tabla $\mathbf{n}^{\mathbf{a}} \mathbf{1}$.Resultados pre test.

\begin{tabular}{cccc}
\hline FASES & $\begin{array}{c}\text { VELOCIDAD } \\
\text { EN KM }\end{array}$ & $\begin{array}{c}\text { DISTANCIA } \\
\text { RECORRIDA (m) }\end{array}$ & $\begin{array}{c}\mathbf{N}^{\mathbf{0}} \text { DE } \\
\text { ESTUDIANTES }\end{array}$ \\
\hline FASE 1 & 8 & 133 & \\
FASE 2 & 9 & 283 & 5 \\
FASE 3 & 9,5 & 441 & 2 \\
FASE 4 & 10 & 608 & 4 \\
FASE 5 & 10,5 & 783 & 2 \\
FASE 6 & 11 & 966 & 8 \\
FASE 7 & 11,5 & 1158 & 6 \\
FASE 8 & 12 & 1358 & 3 \\
FASE 9 & 12,5 & 1566 & 3 \\
FASE 10 & 13 & 1783 & 4 \\
FASE 11 & 13,5 & 2008 & 5 \\
FASE 12 & 14 & 2241 & 5 \\
FASE 13 & 14,5 & 2483 & 3 \\
FASE 14 & 15 & 2733 & 5 \\
FASE 15 & 15,5 & 2991 & 3 \\
FASE 16 & 16 & 3258 & 3 \\
FASE 17 & 16,5 & 3533 & 4 \\
FASE 18 & 17 & 3816 & 3 \\
FASE 19 & 17,5 & 4108 & 0 \\
FASE 20 & 18 & 4408 & \\
FASE 21/23 & 18,5 & 0 & \\
\hline
\end{tabular}

Elaborado por: Grupo de Investigación. 
En esta tabla 1 se presenta los datos en cuanto al número de fases que corresponde recorrer detallando la velocidad en $\mathrm{Km}$ por cada fase, dando un tiempo fraccionado, y la distancia recorrida en metros; a su vez, el número de estudiantes que llegaron a la máxima fatiga, fase en la cual se retiraron para encontrar los datos iniciales sobre el VO2max en cada persona.

Gráfico $N^{o} 1$ Datos de las fases de la aplicación del Pre Test (Course Navette.)

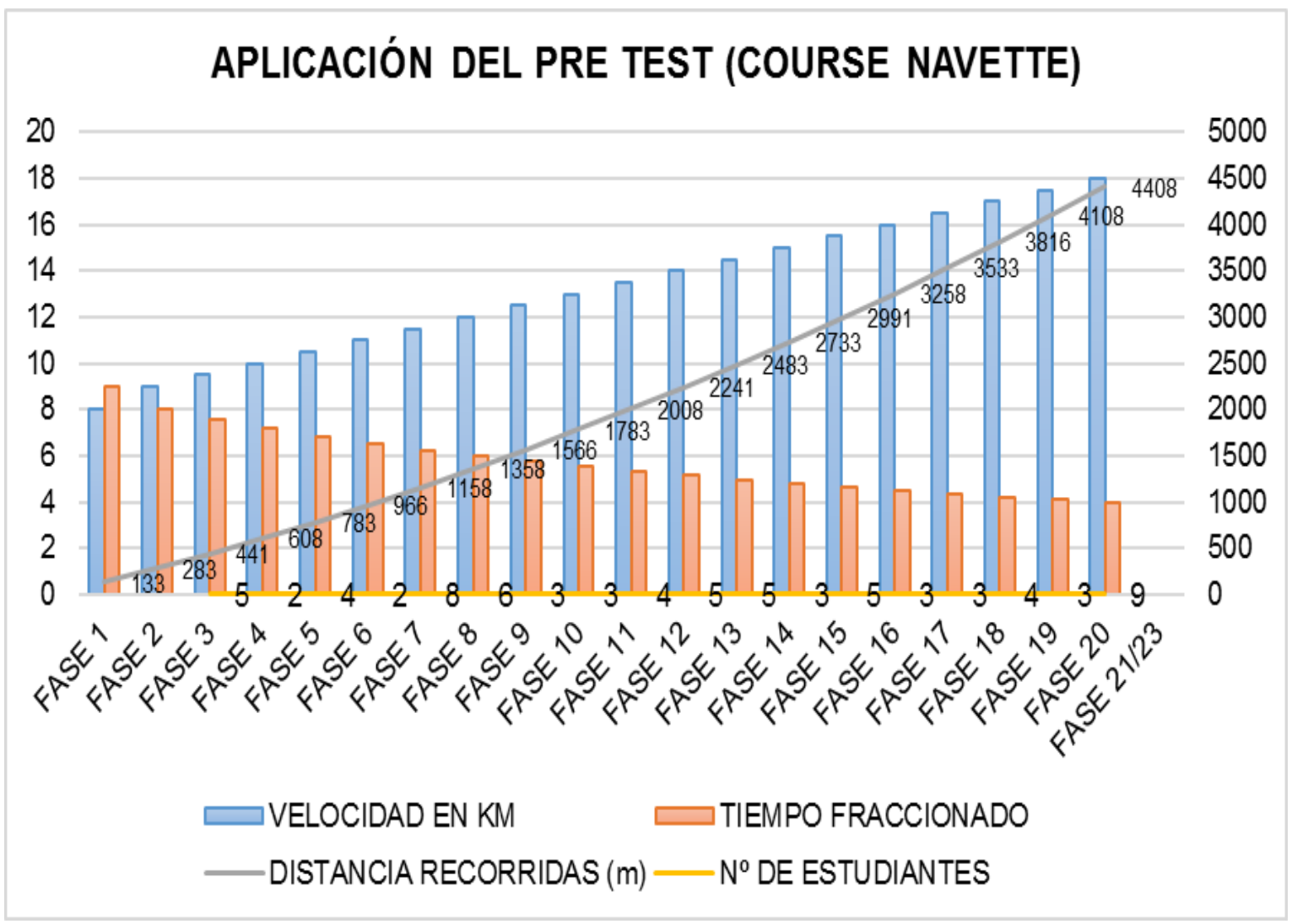

Elaborado por: Grupo de Investigación.

El gráfico 1 relacionado con el pre test aplicado tiene como resultado: de 77 estudiantes que iniciaron la prueba; 9 estudiantes resistieron y llegaron hasta la fase 20, recorriendo una distancia de 4.408 metros y una velocidad de 18 kilómetros; esto quiere decir que, los resultados no fueron alentadores y se aprecia la necesidad de aplicar un programa de juegos pre-deportivos durante las clases de educación física para mejorar el estado físico de los estudiantes. 


\section{Resultados de la aplicación del post test (course navette)}

Tabla $N^{o} 2$ Resultados del Post Test

\begin{tabular}{cccc}
\hline FASES & $\begin{array}{c}\text { VELOCIDAD } \\
\text { EN KM }\end{array}$ & $\begin{array}{c}\text { DISTANCIA } \\
\text { RECORRIDA (m) }\end{array}$ & $\begin{array}{c}\mathbf{N}^{\mathbf{0}} \text { DE } \\
\text { ESTUDIANTES }\end{array}$ \\
\hline FASE 1 & 8 & 133 & \\
FASE 2 & 9 & 283 & \\
FASE 3 & 9,5 & 441 & \\
FASE 4 & 10 & 608 & 5 \\
FASE 5 & 10,5 & 783 & 2 \\
FASE 6 & 11 & 966 & 3 \\
FASE 7 & 11,5 & 1158 & 4 \\
FASE 8 & 12 & 1358 & 2 \\
FASE 9 & 12,5 & 1566 & 2 \\
FASE 10 & 13 & 1783 & 3 \\
FASE 11 & 13,5 & 2008 & 6 \\
FASE 12 & 14 & 2241 & 8 \\
FASE 13 & 14,5 & 2483 & 5 \\
FASE 14 & 15 & 2733 & 4 \\
FASE 15 & 15,5 & 2991 & 5 \\
FASE 16 & 16 & 3258 & 4 \\
FASE 17 & 16,5 & 3533 & 6 \\
FASE 18 & 17 & 3816 & 3 \\
FASE 19 & 17,5 & 4108 & 0 \\
FASE 20 & 18 & 4408 & \\
FASE 21/23 & 18,5 & 0 & \\
\hline
\end{tabular}

Elaborado por: Grupo de Investigación.

En la tabla 2 se observa los resultados en cuanto al número de fases que recorrieron, detallando la velocidad en $\mathrm{Km}$ por cada fase y la distancia recorrida en metros, donde se aprecia el número de estudiantes que llegaron a la máxima fatiga a la que se retiraron, fases en las cuales se retiraron para analizar el incremento sobre el VO2max. 
Gráfico $N^{o} 2$ Datos de las fases de la aplicación del Post Test (Course Navette).

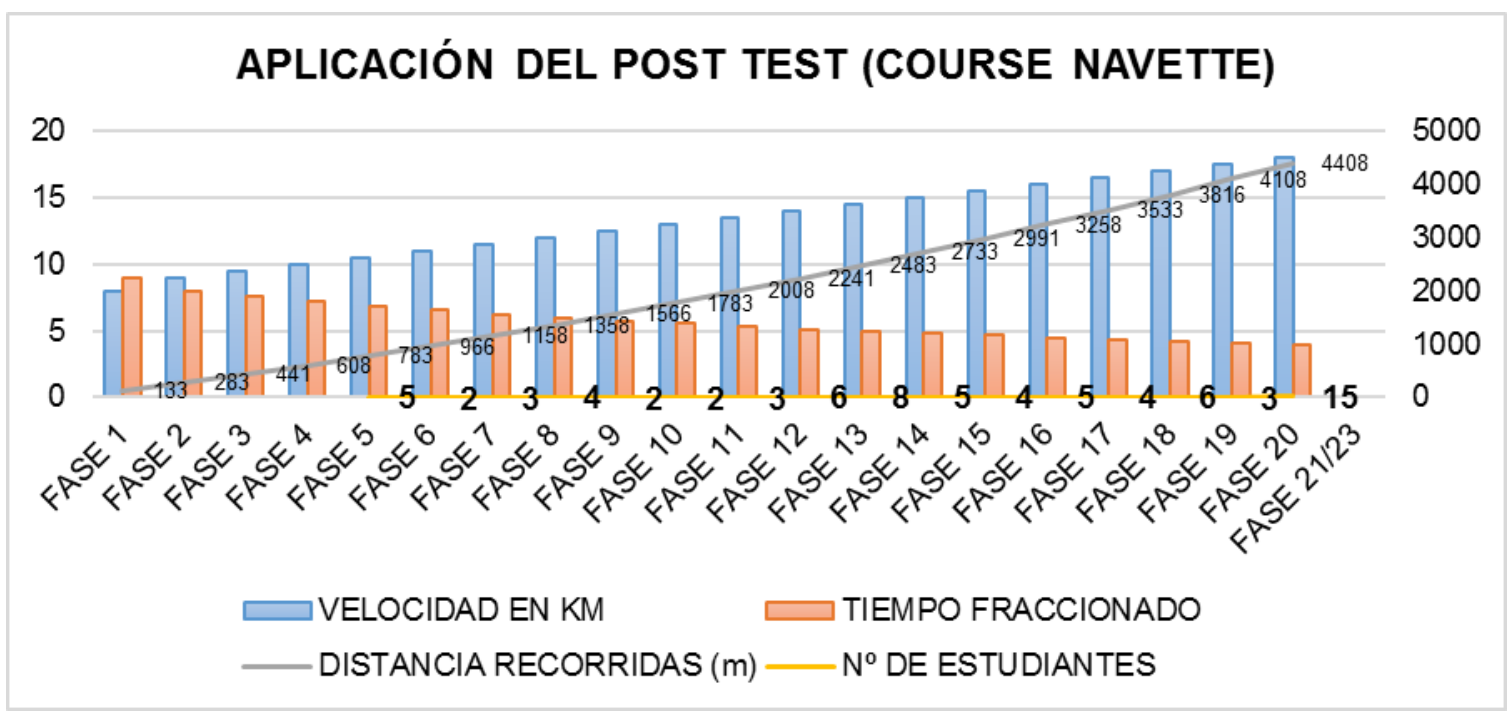

Elaborado por: Grupo de Investigación.

El gráfico 2 relacionado con el post test aplicado tiene como resultado de 77 estudiantes que iniciaron esta prueba; 15 estudiantes resistieron y llegaron hasta la fase 20, recorriendo una distancia de 4.408 metros, con una velocidad de 18 kilómetros; esto quiere decir que los resultados fueron alentadores tras la aplicación del programa de juegos pre-deportivos durante las clases de Educación Física, lo cual muestra una mejora en el estado físico.

Resultados de comparación entre el pre y post test (course navette).

Tabla $N^{a}$ 3. Resultados de comparación entre el pre y post test (Course Navette)

\begin{tabular}{lllll}
\hline \multicolumn{1}{c}{ FASES } & $\begin{array}{c}\text { VELOCIDAD } \\
\text { EN KM }\end{array}$ & $\begin{array}{c}\text { DISTANCIA } \\
\text { RECORRIDAS (m) }\end{array}$ & PRE TEST & $\begin{array}{c}\text { POST } \\
\text { TEST }\end{array}$ \\
\hline FASE 1 & 8 & 133 & & \\
FASE 2 & 9 & 283 & 5 & \\
FASE 3 & 9,5 & 441 & 2 & \\
FASE 4 & 10 & 608 & 4 & 5 \\
FASE 5 & 10,5 & 783 & 2 & 2 \\
FASE 6 & 11 & 966 & 8 & 3 \\
FASE 7 & 11,5 & 1158 & 6 & 4 \\
FASE 8 & 12 & 1358 & 3 & 2 \\
FASE 9 & 12,5 & 1566 & 3 & 2 \\
FASE 10 & 13 & 1783 &
\end{tabular}




\begin{tabular}{lllll} 
FASE 11 & 13,5 & 2008 & 4 & 3 \\
FASE 12 & 14 & 2241 & 5 & 6 \\
FASE 13 & 14,5 & 2483 & 5 & 8 \\
FASE 14 & 15 & 2733 & 3 & 5 \\
FASE 15 & 15,5 & 2991 & 5 & 4 \\
FASE 16 & 16 & 3258 & 3 & 5 \\
FASE 17 & 16,5 & 3533 & 3 & 4 \\
FASE 18 & 17 & 3816 & 4 & 6 \\
FASE 19 & 17,5 & 4108 & 3 & 3 \\
FASE 20 & 18 & 4408 & 9 & 15 \\
FASE 21/23 & 18,5 & 0 & 0 & 0 \\
\hline
\end{tabular}

Elaborado por: Grupo de Investigación.

En la tabla 3 se presenta los resultados entre el pre y post test obtenido acorde al test aplicado a los 77 estudiantes, dando a conocer que en el pre test se tuvo a 9 estudiantes que resistieron hasta la fase 20 y en el post test resistieron 15 estudiantes, con una diferencia de 6 estudiantes, que mejoraron su estado físico llegando a la fatiga máxima y obteniendo resultados favorables.

Gráfico $N^{o} 3$ Resultados de comparación entre el Pre y Post test (Course Navett).

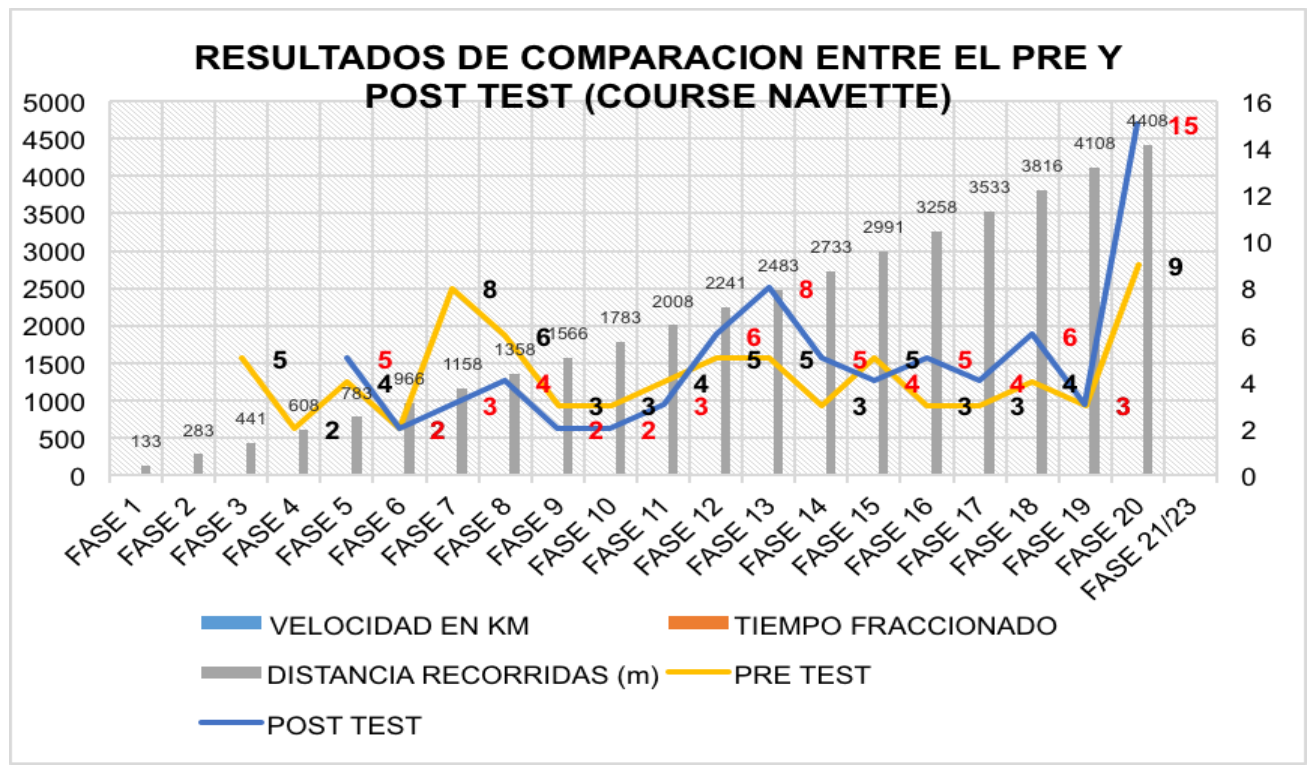

Elaborado por: Grupo de Investigación. 
Análisis: En la gráfica 3 se observa la diferencia que existe entre el pre y post test aplicado a los 77: en cuanto al pre test los estudiantes iniciaron en la fase $1=77$, a partir de la fase 3 se iban retirando y resistieron hasta la fase $20=9$ estudiantes; mientras que para el post test los estudiantes iniciaron en la fase $1=77$, a partir de la fase 5 se iban retirando y resistieron hasta la fase $20=15$ estudiantes teniendo un avance positivo.

Interpretación: La comparación entre el pre test demuestra que, al principio, los estudiantes se encontraban en condiciones físicas bajas y la mayoría se retiraba en las fases iniciales ya que no resistían la demanda de esfuerzo; en cambio, en el post test, hubo una mejoría visible, ya que fueron pocos los estudiantes que en cada fase se iban retirando, y a la fase 20 llegaron 15 estudiantes, en comparación al pre test que solo avanzaron 9 estudiantes; concluyendo así que, al aplicar el programa de juegos pre-deportivos de manera progresiva ayuda a mejorar el consumo de oxígeno y el incremento en la condición física.

\section{Comprobación de la hipótesis.}

Resultados según la prueba de kolmogorov - smirnov para una muestra.

A continuación, se describe los resultados obtenidos del antes y después según la prueba de Kolmogorov-Smirnov para una muestra, tras la aplicación del test de Course Navette.

La tabla 4 muestra los estadísticos descriptivos de los participantes.

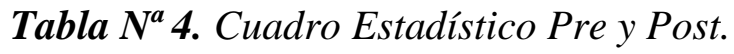

\begin{tabular}{llll}
\hline \multicolumn{2}{ll}{ Estadísticos } & & \\
\hline \multirow{2}{*}{ N } & Válidos & Pre & Post \\
& Perdidos & 0 & 77 \\
&
\end{tabular}

Elaborado por: Grupo de Investigación.

Posteriormente en la tabla 5 se muestra el estadístico de correlación para los datos del antes y después, con un intervalo de confianza del $95 \%$

Tabla $N^{a} 5$ Prueba de muestras relacionadas.

\begin{tabular}{lllll}
\hline \multicolumn{4}{l}{ Prueba de muestras relacionadas } \\
\hline & & $\mathrm{T}$ & $\mathrm{gl}$ & Sig. (bilateral) \\
Par & post - & 16,83 & 76 &, 000 \\
1 & pre & 2 & & \\
\hline
\end{tabular}

Elaborado por: Grupo de Investigación.

Finalmente, en la tabla 6 y 7 se observa la correlación existente entre el pre y post test. 
Tabla $N^{a}$ 6. Resultados según la prueba de Kolmogorov-Smirnov.

\begin{tabular}{|c|c|c|c|}
\hline \multicolumn{4}{|c|}{ Prueba de Kolmogorov-Smirnov para una muestra } \\
\hline \multirow{2}{*}{\multicolumn{2}{|c|}{ 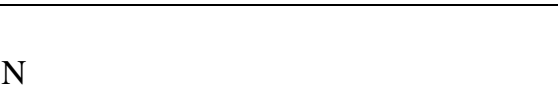 }} & Pre & Post \\
\hline & & 77 & 77 \\
\hline \multirow[t]{2}{*}{ Parámetros normales ${ }^{\mathrm{a}, \mathrm{b}}$} & Media & 2316,66 & 2833,45 \\
\hline & $\begin{array}{l}\text { Desviación } \\
\text { típica }\end{array}$ & 1275,819 & 1183,997 \\
\hline \multirow{3}{*}{$\begin{array}{l}\text { Diferencias } \\
\text { extremas }\end{array}$} & Absoluta & ,124 & ,108 \\
\hline & Positiva &, 124 & ,092 \\
\hline & Negativa &,- 088 &,- 108 \\
\hline \multicolumn{2}{|c|}{ Z de Kolmogorov-Smirnov } & 1,092 & ,951 \\
\hline \multicolumn{2}{|l|}{ Sig. asintót. (bilateral) } & 184 & 326 \\
\hline
\end{tabular}

Elaborado por: Grupo de Investigación.

Tabla $N^{a}$ 7. Correlaciones de muestras relacionadas.

\begin{tabular}{lllll}
\hline \multicolumn{5}{c}{ Correlaciones de muestras relacionadas } \\
\hline \multicolumn{7}{c}{ Par 1} & post y pre & 77 & Correlación & Sig. \\
& & 979 &, 000 \\
\hline
\end{tabular}

Elaborado por: Grupo de Investigación.

Consecutivamente, se evaluó si la intervención muestra mejoras significativas o no en la condición física de los participantes. Para lo cual se usó la prueba $t$ para muestras relacionadas; en primer lugar, se estableció la normalidad de los datos tanto del pre y post test, con lo cual se cotejó el comportamiento del antes y el después, es decir, los datos cumple con los supuestos de normalidad para ambos casos, cuyo $\mathrm{p}$ valor es mayor que 0,05 ; por lo tanto, los datos de la población de estudio se encuentran normalmente distribuidos, y se aplicar una prueba t para muestras relacionadas para poder comparar los resultados pre y post.

En tal sentido, el p valor obtenido es de 0,000, es decir, existe diferencias significativas entre el antes y el después, por consiguiente, los valores luego del programa presentan valores significativos en la distancia recorrida tras la aplicación de la intervención realizada, cuya Sig. (bilateral) es de ,000. Con lo cual se concluye que la intervención mejoró las puntuaciones sobre el VO2max en los participantes del estudio.

\section{Conclusiones.}

- Consideramos que el test de Course Navette es una prueba confiable y sencilla para poder analizar los resultados sobre el VO2MAX y la condición física de las personas que practican deportes o realizan actividad física de forma regular.

- En cuanto al programa de intervención, se puede decir que se pudo efectuar de manera eficiente, ya que permitió medir el consumo de oxígeno máximo, pues los 
resultados obtenidos responden a una planificación estructurada en función de juegos pre-deportivos, los cuales deben ser ejecutados continuamente para que los jóvenes desarrollen su capacidad aeróbica, cuyo disfrute personal refleja los resultados obtenidos.

- La comprobación de la hipótesis de trabajo muestra claramente los resultados de la aplicación y los efectos que el programa de intervención en el VO2MAX incrementó el consumo máximo de oxígeno de los adolescentes, es decir, se cumplieron los parámetros establecidos para este tipo de prueba, teniendo el valor $p$ que da 0,000, por ello, existen diferencias significativas tras la aplicación del programa de intervención.

- Al elaborar un programa de juegos pre-deportivos se fomenta las diferentes actividades físico-recreativas con la finalidad de incentivar a los adolescentes a mejorar y potencializar su condición física. Los datos obtenidos en el presente estudio pueden ser de gran utilidad para futuras investigaciones, relacionadas con el campo del entrenamiento deportivo y el análisis de la condición física en adolescentes deportistas.

\section{Referencias bibliográficas.}

Arévalo-Sánchez, X. (enero-junio de 2016). Los juegos cooperativos como alternativa para generar paz. Ra Ximhai, 12(3), 335-343. Obtenido de http://www.redalyc.org/articulo.oa?id=46146811023

Cabrera Hernández, D., \& Cruz Vadillo, R. (Enero-Junio de 2016). Reforma educativa como cambio curricular: representaciones de docentes en una escuela primaria. CPU-e, Revista de Investigación Educativa(22), 200-225. Obtenido de http://www.redalyc.org/articulo.oa? $\mathrm{id}=283143550010$

Casas, A. G.-C.-S. (2015). Capacidad aeróbica y calidad de vida en escolares de 8 a 12 años. Clínica e Investigación en Arteriosclerosis, 27(5), 239-245. Obtenido de https://dialnet.unirioja.es/servlet/articulo?codigo $=5210030$

Delgado Noguera, M. Á. (Julio-Diciembre de 2015). Los estilos de enseñanza de la Educación Física y el Deporte a través de 40 años de vida profesional. RETOS. Nuevas Tendencias en Educación Física, Deporte y Recreación(28), 240-247. Obtenido de http://www.redalyc.org/pdf/3457/345741428043.pdf

Díaz, D. A. (2015). Tiempo límite a la velocidad asociada al consumo máximo de oxígeno en jóvenes atletas del municipio de Chía, Colombia. Lecturas: Educación física y deportes(206). Obtenido de https://dialnet.unirioja.es/servlet/articulo?codigo $=5289113$

Díaz, R. M. (2016). Los juegos cooperativos y su incidencia en los estados de ánimo y las emociones en escolares de 10-12 años. EmásF: revista digital de educación 
física(41), 108-132. Obtenido de file:///D:/Descargas/DialnetLosJuegosCooperativos YSuIncidenciaEnLosEstadosDeAn-5558017.pdf

Figueroa, J. A. (2015). Relación del VO2máx y la masa muscular de tudiantes de educación física, deporte y recreación. Lecturas: Educación física y deportes(207). Obtenido de file:///C:/Users/9/Downloads/Dialnet-

RelacionDelVO2max YLaMasaMuscularDeTudiantesDeEduca-5391078.pdf

García, G. C. (2014). Test course navette de 20 metros con etapas de un minuto. Una idea original que perdura hace 30 años. Apunts. Medicina de l'Esport, 49(183), 93-103. Obtenido de http://appswl.elsevier.es/watermark/ctl_servlet?_f=10\&pident_articulo=90349201\& pident_usuario $=0 \&$ pcontactid $=\&$ pident_revista $=277 \&$ ty $=154 \&$ accion $=$ L\&origen $=b$ ronco\%20\&web=www.apunts.org\&lan=es\&fichero=277v49n183a90349201pdf001. pdf\&anuncioPdf=ERROR_publi_pdf

Gómez, C. N. (2017). La importancia del juego como función social a través del desarrollo del ser humano. Ciencia y actividad fisica.

González, J. G.-M. (2016). Personalidad y estilos de toma de decisiones en la práctica deportiva. Revista Iberoamericana de Psicología del Ejercicio y el deporte, 11(1), 107-112. Obtenido de http://www.redalyc.org/articulo.oa?id=311143051012

Granja, F. A. (2016). Influencia de los juegos predeportivos en la preparación teórica y técnicotáctica de karatecas infantiles. Lecturas: Educación Física y Deportes(223). Obtenido de file:///D:/Descargas/karatecasinfantiles.pdf

Graña, V. A. (2015). Los juegos populares como herramienta para la convivencia en los recreos. Revista de estudios e investigacion en psicologia y educacion(2), 1-5. Obtenido de http://revistas.udc.es/index.php/reipe/article/view/353

Longo, A. F. (2017). Valoración del VO 2 max en deportistas: valora un riguroso estudio comparativo de métodos entre el test Yo-Yo y la medición directa. Apunts: Medicina de l'esport, 52(193). Obtenido de http://www.apunts.org/es/valoraciondel-vo-2max-deportistas/articulo/90460860/

BIBLIOGRAPHY Lucio, N. D. (2018). Accuracy of Fitbit Charge 2 at Estimating VO2max, Calories, and Steps on a Treadmill. In International Journal of Exercise Science: Conference Proceedings , 2(10), 11.

Martinez López, E. (2004). Aplicación de la prueba cooper, course navette y test de ruffier. resultados y análisis estadístico en educación secundaria. Revista Internacional de Medicina y Ciencias de la Actividad Física y el Deporte, 4(15), 163-182. Obtenido de http://cdeporte.rediris.es/revista/revista15/artcooper.htm

Miranda, M. D. (2016). Juegos pre-deportivos de bádminton para el deporte para todos. PODIUM-Revista de Ciencia y Tecnología en la Cultura Física, 11(3), 167-175. Obtenido de http://podium.upr.edu.cu/index.php/podium/article/view/672/pdf 
Morales, D. A. (Junio de 2017). Influencia de los juegos predeportivos sobre el rendimiento técnico y motriz del voleibol de iniciación. Lecturas: Educación Física y Deportes(229), 1. Obtenido de http://www.efdeportes.com/efd229/influencia-de-losjuegos-predeportivos-del-voleibol.htm

Polo-Benítez, J. \&.-S. (2017). Revisión del efecto del entrenamiento de alta intensidad sobre el rendimiento físico en futbolistas. SPORT TK-Revista EuroAmericana de Ciencias del Deporte, 6(2), 31-38. Obtenido de https://dialnet.unirioja.es/servlet/articulo?codigo $=6102003$

Pumar, B. (2015). Efectos de un programa de actividad física en escolares. scielo, 17(2). Obtenido de http://www.scielo.org.ar/scielo.php?script=sci_arttext\&pid=S231425612015000200001

Ruitón, O. M. (2015). Juegos lúdicos para la mejora de la motricidad gruesa. In Crescendo Educación y Humanidades, 2(2), 1-10. Obtenido de http://revistas.uladech.edu.pe/index.php/increscendoeducacion/article/view/1041/860

Ruíz, H. A. (2015). Comportamiento de los niveles del vo2 máximo en futbolistas prejuveniles en diferentes altitudes. Revista Digital: Actividad Física y Deporte, 1(2). Obtenido de file:///C:/Users/9/Downloads/7-16-1-SM.pdf

Valarezo Mendoza, E. V. (2017). Programa de actividades físico-recreativas para desarrollar habilidades motrices en personas con discapacidad intelectual. Revista Cubana de Investigaciones Biomédicas, 36(1), 1-13. Obtenido de http://scielo.sld.cu/pdf/ibi/v36n1/ibi08117.pdf

Velásquez, J. C. (mayo-agosto de 2015). ¿Puede la frecuencia cardíaca ser un estimador del consumo de oxígeno para segmentos corporales?. Revista de la Universidad Industrial de Santander, 47(2), 159-168. Obtenido de http://www.redalyc.org/articulo.oa?id=343839278007

Véliz, C. V. (2016). Efectos de un entrenamiento interválico de alta intensidad (hiit) sobre el vo2máx y la recuperación intermitente en jóvenes nadadores. EmásF: revista digital de educación física(39), 48-57. Obtenido de file:///C:/Users/9/Downloads/Dialnet-

EfectosDeUnEntrenamientoIntervalicoDeAltaIntensida-5370985.pdf

Yanelis Cobas-Ortiz, E. I.-N.-C. (Abril-Junio de 2015). La caracterización psicosocial del atleta del deporte Atletismo. Una premisa en la iniciación deportiva. EduSol, 15(51), 89-99. Obtenido de http://www.redalyc.org/html/4757/475747192007/

Yépez, M. M. M., \& Benítez, J. E. M. (2017). Estrategias metodológicas para la gimnasia aeróbica en las clases de educación física. EsmásF Revista Digital de Educación Física(46), 91. Obtenido de file:///D:/Descargas/DialnetEstrategiasMetodologicasParaLaGimnasiaAerobicaEnLa-5963363.pdf 
Para citar el artículo indexado.

Mocha J., Garcés S., Medina S., Castro W., \& Suntasig M. (2018). Análisis del consumo máximo de oxígeno (vo2máx) post intervención de un programa en juegos pre-deportivos. Revista electrónica Ciencia Digital 2(2), 232-247. Recuperado desde:

http://cienciadigital.org/revistacienciadigital2/index.php/CienciaDigital/article/view/85/79

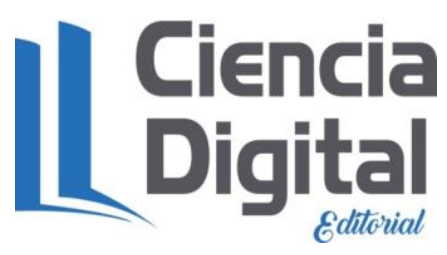

El artículo que se publica es de exclusiva responsabilidad de los autores y no necesariamente reflejan el pensamiento de la Revista Ciencia Digital.

El articulo queda en propiedad de la revista y, por tanto, su publicación parcial y/o total en otro medio tiene que ser autorizado por el director de la Revista Ciencia Digital.
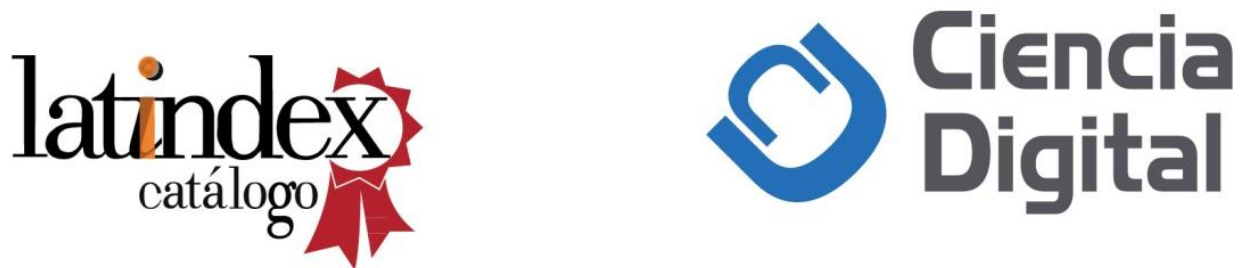